\title{
O DESENVOLVIMENTO DA COMPETÊNCIA BILINGUE DE CRIANÇAS EM IDADE PRÉ-ESCOLAR
}

\author{
Anabela Fernandes Custódio \\ Universidad de Extremadura anabelacustodio@gmail.com \\ Inmaculada Sanchez Casado \\ Universidad de Extremadura iscasado@unex.es
}

Recepción Artículo: 22 octubre 2021 Admisión Evaluación: 22 octubre 2021 Informe Evaluador 1: 23 octubre 2021 Informe Evaluador 2: 24 octubre 2021

Aprobación Publicación: 24 octubre 2021

\section{RESUMEN}

0 ambiente linguístico e cultural é determinante para o desenvolvimento de uma língua, particularmente para a criança bilingue. Se o contexto é favorável ao bilinguismo, a aquisição de uma outra língua torna-se uma competência positiva na vida da criança. Para as crianças que iniciam a aprendizagem escolar no seu país de origem ou cujos pais têm como língua familiar o português, termos como "em cima, cheio, ao lado, muitos, Ionge", entre outros, designados por conceitos básicos, são adquiridos naturalmente. Nas crianças bilingues em que uma língua é menos falada, no caso do presente estudo, o português, pode surgir um défice de compreensão destes conceitos, o qual, eventualmente, pode comprometer o processo de aprendizagem futura. A inclusão de crianças numa sociedade distinta da sua, leva a que estas tenham de se adaptar a modificações sociais e linguísticas e a língua familiar pode vir a ser trocada pela língua oficial do país. Tendo em conta estas vivências, este estudo desenvolve-se com crianças em idade pré-escolar que vivem numa comunidade linguística onde estão expostas ao português e francês desde cedo. Tem como objetivo avaliar a compreensão de conceitos básicos em português, em crianças com idades compreendidas entre os quatro e os cinco anos, através da aplicação do Teste de Conceitos Básicos de Boehm Pré-Escolar 3 (BTCB). 0 processo avaliativo desenvolve- se com dois grupos A e B e tem três

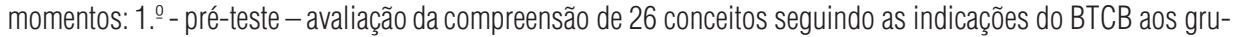
pos A e B; $2 .{ }^{\circ}$ - jogos infantis - implementação no grupo $A ; 3 .{ }^{\circ}$ - pós-teste - aplicação do BTBC aos grupos A e $B$ relativamente às questões não conseguidas no pré-teste. Após a análise de dados, constituiu-se um conjunto informativo sobre as competências conceptuais das crianças, o desenvolvimento cognitivo e o papel das variáveis na compreensão dos conceitos.

Palabras clave: bilinguismo; conceitos básicos; jogos infantis; teste de Boehm

\section{ABSTRACT}

The development of bilingual competence in preschool children. The linguistic and cultural environment is crucial for the development of a language, particularly for the bilingual child. If the context is favorable 


\section{O DESENVOLVIMENTO DA COMPETÊNCIA BILINGUE DE CRIANÇAS EM IDADE PRÉ-ESCOLAR}

to bilingualism, the acquisition of another language becomes a positive skill in the child's life. For children who start school learning in their country of origin or whose parents have Portuguese as their familiar language, terms such as "above, full, beside, many, far", among others, designated by basic concepts, are naturally acquired . In bilingual children whose language is less widely spoken, in the case of this study, Portuguese, there may be a deficit in understanding these concepts, which may eventually compromise the process of future learning. The inclusion of children in a society different from theirs means that they have to adapt to social and linguistic changes and the familiar language can be changed to the official language of the country. Taking these experiences into account, this study is developed with preschool-age children who live in a linguistic community where they are exposed to Portuguese and French from an early age. It aims to assess the understanding of basic concepts in Portuguese, in children aged between four and five years, through the application of the Boehm Pre-School Basic Concepts Test 3 (BTCB). The evaluation process is developed with two groups A and B and has three stages: 1st - pre-test - assessment of the understanding of 26 concepts following the indications of the BTCB for groups A and B; 2nd - children's games - implementation in group A; 3rd - post-test

- application of the BTBC to groups A and B regarding questions not achieved in the pre- test. After data analysis, an informative set was created on the conceptual skills of children, cognitive development and the role of variables in understanding the concepts.

Keywords: bilingualism; basic concepts; Boehm test; children's games

\section{INTRODUCCIÓN}

0 bilinguismo infantil é um tema atual sobre 0 qual se têm debruçado estudiosos como Flores (2019), Grosjean (2017), Abdelilah-Bauer (2015), entre outros. Flores (2019) destaca os estudos psicolinguísticos que analisam 0 desenvolvimento neuropsicológico e cognitivo desenvolvido pela psicóloga Ellen Bialystok que evidenciam os resultados positivos do bilinguismo sobre funções cognitivas e linguísticas. A autora refere ainda que as crianças são capazes de adquirir duas línguas na infância sem esforço cognitivo adicional. Devido à diversidade de contextos em que a aquisição bilingue ocorre, alguns estudos (Cote \& Bornstein, 2014; Hoff et al., 2012; Marchman, Martínez-Sussman \& Dale, 2004; 2012) têm procurado identificar fatores intervenientes no processo de desenvolvimento da competência lexical bilingue. Neste domínio, fatores como a quantidade e a qualidade da exposição linguística, entre outros, têm sido apresentados como variáveis preditivas do desenvolvimento lexical de crianças bilingues (Correia \& Flores, 2017; Unsworth, 2016). Nas crianças bilingues em que uma língua é menos falada, no caso do presente estudo, o português, pode surgir um défice de compreensão destes conceitos, o qual, eventualmente, pode comprometer o processo de aprendizagemfutura.

\section{OBJETIVOS DE LA INVESTIGACIÓN}

0 objetivo geral do estudo é estabelecer se a relação entre os conceitos básicos de Boehm e os jogos infantis favorece 0 desenvolvimento cognitivo. Os objetivos específicos definidos são: identificar se há diferenças significativas nos resultados do pré-teste dos grupos A e B na faixa etária dos quatro anos; identificar se há diferenças significativas nos resultados do pré-teste dos grupos A e B na faixa etária dos cinco anos; identificar se há diferenças significativas nos resultados do pós-teste dos grupos $A$ e $B$ na faixa etária dos quatro anos; identificar se há diferenças significativas nos resultados do pós-teste dos grupos A e B na faixa etária dos cinco anos; comparar os resultados do pré-teste, jogos infantis e do pós-teste no grupo A; comparar os resultados do pré-teste e do pós-teste no grupo B; verificar que variáveis podem influenciar o desempenho das crianças na compreensão dos conceitos básicos; averiguar se os jogos infantis influenciam a compreensão dos conceitos básicos em crianças de quatro e cinco anos de idade e; identificar o grau de dificuldade entre conceitos.

\section{MUESTRA Y/O PARTICIPANTES}

As crianças participantes neste estudo são lusodescendentes que residem em França e frequentam o préescolar que se divide em três secções: pequena secção - 3 anos (PS); média secção - 4 anos (MS) e; grande sec- 
ção - 5 anos (GS). As crianças começam por frequentar a pequena secção, onde aprendem apenas francês, na escola francesa. Posteriormente, passam às secções seguintes (média e grande) e frequentam paralelamente uma classe de francês e outra de português, isto porque na secção portuguesa existem apenas dois níveis de escolaridade no pré-escolar: média secção e grande secção. As 31 crianças bilingues participantes do estudo estão inseridas em duas classes do pré-escolar (grupo A e B) e a frequência nas aulas de português é idêntica. As classes referidas como grupos A e B foram testadas na escola onde aprendem o português. 0 grupo A, no Liceu Internacional e 0 grupo B na Escola Normandie Niémen, um dos polos da secção portuguesa. As avaliações do pré-teste, jogos infantis e pós-teste decorreram nos horários habituais das aulas de português e foram postas em prática pelas professoras das respetivas classes. Todos os participantes crescem e vivem num ambiente bilingue, no qual o português é usado em contexto doméstico e o francês é a língua maioritária, ou seja, a língua de escolarização e da sociedade.

\section{METODOLOGÍA Y/O INSTRUMENTOS UTILIZADOS}

Este estudo apoia-se numa metodologia quantitativa que segue uma abordagem fundamentada no empirismo. Como descrito por Carmo \& Ferreira (2015, p. 115), 0 investigador deve procurar o conhecimento nos factos reais da sua investigação pondo de parte toda as informações desnecessárias à pesquisa. Na abordagem quantitativa, a recolha de dados realiza-se com instrumentos que já foram utilizados em estudos anteriores e mostraram ser fiáveis. A observação, a medição e a documentação das variáveis suportam a recolha de dados numa pesquisa quantitativa. Sampieri, Collado e Lucio (2013) defendem que a abordagem quantitativa pode ser considerada um percurso metodológico sequencial e compensativo, que se desenvolve em uma sequência rigorosa de fases, iniciando-se num problema delimitado e concreto e com a formulação de questões específicas. De acordo com 0 mencionado, este estudo procura caracterizar, descrever e interpretar para explicar os dados analisados. Para objetivar o proposto por esta investigação aplicou-se uma metodologia também qualitativa porque do ponto de vista de Gonçalves et al. (2021) a abordagem qualitativa pretende dar sentido à experiência humana. É consensual que numa investigação pedagógica, o investigador utilize a abordagem qualitativa pois pretende otimizar a sua prática. A investigação qualitativa considera-se como um tipo de investigação que tem como fonte direta de dados 0 ambiente natural. É uma abordagem que favorece mais o processo que os resultados ou produtos e em que os dados recolhidos e os resultados são descritos minuciosamente. Tem de haver colaboração entre investigador e participantes. Algumas características desta investigação são referidas por Gómez, Flores e Jiménez (1999); Stake (2005); Bogdan e Biklen (2010). 0 desenvolvimento deste estudo baseou-se na aplicação de um teste de conceitos básicos através do teste norte americano: Boehm Test of Basic Concepts - Third Edition (BTBC), e na participação em jogos didáticos infantis elaborados com o propósito de ajudar as crianças a compreender os conceitos básicos e impulsionar o sistema cognitivo. 0 Teste de Conceitos Básicos de Boehm Pré-Escolar 3 (BTCB) é um teste que representa os conceitos em imagens. 0 professor lê a palavra que designa 0 conceito e a criança tem de escolher a imagem referente a esse conceito, de entre as imagens que lhe são apresentadas. Os jogos infantis são fáceis, elaborados com material reciclado ou material existente nos espaços em que eles decorrem (sala de aula e salão de jogos) e consistem na descoberta de um conceito através da seleção do objeto correto que representa 0 conceito pretendido.

\section{RESULTADOS ALCANZADOS}

No pré-teste e pós-teste foi seguida a ordem em que os conceitos são apresentados no caderno de figuras. No pré-teste (GA e GB) são avaliados 26 conceitos e esperados 52 acertos (respostas corretas) que correspondem a 100\%. Por último, no pós-teste, as crianças são avaliadas nos conceitos que erram no pré-teste e são esperados 52 acertos (respostas corretas) que corresponde a 100\%. Para a análise estatística optou-se por selecionar do pré-teste e do pós-teste os conceitos que foram trabalhados nos jogos infantis para ter um termo de comparação. Durante a aplicação do teste Boehm (2012) e seguindo as indicações do mesmo as respostas incorretas foram identificadas pelo valor 0 e as corretas com valor 1 , as respostas dadas como antónimos também são iden- 


\section{O DESENVOLVIMENTO DA COMPETÊNCIA BILINGUE DE CRIANCAS EM IDADE PRÉ-ESCOLAR}

tificadas pelo valor 0 . Considerando cada conceito avaliado, foram calculadas a frequência absoluta e a frequência relativa de respostas corretas para cada grupo e para o total dos sujeitos incluídos na pesquisa. Ao analisar os resultados obtidos no grupo A/MS ( $n=7)$, no pré-teste verifica-se que a pontuação direta, que corresponde aos acertos, varia entre os 0 (0\%) e os 48 acertos (92\%). 0 número total de acertos do teste são 52 (100\%). As crianças erraram os conceitos: "o mais perto", "0 maior" (5x); "na frente", "ao redor" (6x); "antes", entre (7x); "0 mais curto", "meio" (8x); "alguns, mas não muitos" (9x); "último", "menos" (10x); "o mais longe" (11x). Os conceitos que mais erraram pertencem ao domínio espaço (8 conceitos) e ao domínio quantidade (4 conceitos). Selecionaram a imagem representando o antónimo para os conceitos nos domínios: espaço - "o mais perto", "0 mais longe", "o mais baixo", "antes", "último", "meio"; quantidade - "alguns, mas não muitos", "o mais curto" e "menos"; e no domínio outros, para o conceito "diferente". Os resultados melhoraram no pós-teste: duas crianças acertaram todos os conceitos, uma falhou o conceito "entre", três falharam, 6, 7, e 10 conceitos. Uma criança não acertou em nenhum. No grupo A/GS (n=12), no pré-teste, as crianças erraram os seguintes conceitos: "terminado", "ambos" (1x); "o mais comprido", "atravessar", "muitos", "igual", "inferior", "meio" (2x); "o menor", "ao redor", "0 mais perto", "o mais alto", "mais", "0 mais baixo", "juntos", "menos", "primeiro" (3x); "na frente", "0 maior", "entre" (4x); "0 mais longe", "diferente"(5x); "último", "0 mais longe" (6x); "0 mais curto", "alguns, mas não muitos" (9x); "antes"(11x). Os conceitos que as crianças mais erraram pertencem ao domínio espaço (11 conceitos) e ao domínio quantidade (7 conceitos). Utilizaram o antónimo para os conceitos inseridos nos seguintes domínios: espaço - "o mais longe", "último"; quantidade - "o mais alto", "o maior", "o menor", "mais"; outros: "diferente". Neste grupo, a pontuação direta, que corresponde aos acertos, varia entre 3 (6\%) e 51 acertos (98\%). Relativamente aos resultados obtidos pelas crianças no pós-teste conclui-se que no grupo A/GS ( $n=12)$ sete crianças acertaram em todos os conceitos (100\%) duas crianças tiveram 51 acertos cada (98\%); uma criança atingiu 49 acertos (96\%). De referir, duas crianças que evoluíram do pré para o pós-teste, mas ainda erraram 14 conceitos e 19 conceitos respetivamente. No grupo B/MS (n=8), no pré-teste verifica-se que a pontuação direta varia entre os 14 (27\%) e os 36 acertos (69\%). As crianças erraram com muita frequência os seguintes conceitos: "terminado", "o mais baixo" "mais", "o maior" (5x); "atravessar", "diferente", "o mais comprido" (6x); "na frente" (7x); "muitos", "antes" (8x); "0 mais longe" (9x); "ao redor", "menos" (10x); "o mais curto", "entre" (12x); "igual", "meio" (13x); "juntos", "alguns, mas não muitos" (14x); "último" (15x). Os conceitos que as crianças erraram com mais frequência pertencem ao domínio espaço. Utilizaram o antónimo para os conceitos nos domínios: espaço "o mais perto", "atravessar", "ao redor", antes", "o mais longe", "o mais baixo", "último", "inferior", "juntos", "primeiro", "entre"; quantidade - "0 menor", "0 mais comprido", "o mais alto", "muito", "mais", "o mais curto", "alguns, mas não muitos" e "menos"; tempo - "terminado"; e outros - "diferente". No pós-teste, as crianças atingiram a pontuação máxima (100\%) nos conceitos "o mais perto", "0 mais comprido", "primeiro", "igual", "0 maior" e "ambos". No grupo B/GS ( $n=4)$, no pré-teste duas crianças tiveram um bom resultado, outra errou cinco conceitos e a última 30. No pós-teste, três crianças acertaram todos os conceitos (100\%) e a quarta também evoluiu, acertou em 46 conceitos (88\%). Nos jogos infantis, a aplicação dos conceitos teve em atenção os resultados obtidos no pré-teste pelas crianças do grupo A. Foram selecionados 19 conceitos dos 26 apresentados no teste. Foi trabalhado um jogo para cada conceito, realizando-se assim 19 jogos no total. Os jogos infantis só foram aplicados ao grupo A. 0 grupo B teve neste estudo o papel de grupo de controlo. Apresenta-se seguidamente uma descrição sobre os acertos dos conceitos pelas crianças que participaram nos jogos infantis. No grupo A/MS $(n=7)$ - três crianças desta faixa etária na participação dos jogos compreenderam o conceito "mais" e duas o conceito "igual"; para oito crianças os jogos ajudaram na compreensão dos conceitos "o menor" e "ao redor"; cinco crianças compreenderam os conceitos "o mais perto", "o mais longe", "o mais comprido", "alguns, mas não muitos", "o mais baixo", e "último"; duas os conceitos "na frente", "maior", "menos" e "ambos"; onze crianças bilingues compreenderam os conceitos "juntos", "diferente", "primeiro" e "o mais alto"; sete crianças compreenderam o conceito "terminado." Nesta faixa etária, os meninos revelam uma melhor compreensão em dezasseis conceitos do que as meninas. Quantoao grupo A/GS ( $n=12$ ) - na participação dos jogos, 7 crianças desta faixa etária com- 
preenderam o conceito "ao redor". Os jogos ajudaram nove crianças na compreensão dos conceitos "o menor", "alguns, mas não muitos", "igual" e "último"; dez crianças compreenderam através dos jogos os conceitos "0 mais perto", "o mais longe"; "terminado", "o mais comprido", "na frente", "ambos"; "primeiro"; "o mais baixo"; onze crianças compreenderam os conceitos "diferente", "o mais alto", "maior" e "menos"; doze crianças compreenderam o conceito "juntos"; onze crianças compreenderam o conceito "mais". Nesta faixa etária, as meninas revelam uma melhor compreensão em doze conceitos do que os meninos. No caso dos jogos, a frequência diminuiu, pois, algumas crianças não participaram por estarem ausentes. Como não houve possibilidade de flexibilizar o tempo e repetir os jogos, algumas crianças não beneficiaram desta atividade lúdica. Observando os resultados e os conceitos que as crianças mais erraram no pré-teste e os resultados obtidos no pós-teste pelo grupo A tanto na faixa dos quatro anos como na dos cinco anos, conclui-se que a aprendizagem de conceitos em atividades lúdicas nomeadamente os jogos, ajudam as crianças a compreenderem melhor os conceitos porque através da implementação desta atividade lúdica tiveram a oportunidade de concretizar na prática os seus conhecimentos relativamente aos conceitos. Os jogos ajudam visualmente a consolidar a ideia que já têm dos conceitos. A aprendizagem em grupo pode ajudar uma criança tímida. No caso deste estudo, foi o que aconteceu. Um menino não colaborou no pré-teste nem no pós-teste, mas compreendeu a maior parte dos conceitos trabalhados com os jogos. Como se demonstra pelos resultados obtidos pelo grupo B no pré e pós-teste, as crianças são capazes de aprender nos mais diversos contextos e espaços onde possam interagir e fazer as suas próprias descobertas. A sua capacidade de aprendizagem depende dos estímulos que recebem para construir o seu próprio conhecimento.

\section{DISCUSIÓN}

As análises estatísticas revelam que não há diferenças significativas dentro dos dois grupos e respetivas idades quanto à compreensão dos conceitos avaliados. No entanto, os resultados obtidos pelo grupo A, quatro anos, no pré-teste são mais positivos que os resultados alcançados pelo grupo B no pré-teste na mesma faixa etária. A intervenção com os jogos infantis não teve relevância no desempenho final a nível cognitivo dos falantes bilingues do grupo A porque eles alcançaram bons resultados no pré-teste, tendo pouca margem de progressão. Porém, no grupo A (MS e GS) houve uma evolução estatisticamente significativa do pré para o pós-teste, sendo a magnitude da diferença elevada em ambos, embora seja mais alta na MS. No grupo B/MS houve uma evolução significativa do pré para o pós-teste, sendo a magnitude da diferença considerada elevada. No mesmo grupo, mas na GS, embora tenha havido uma melhoria do pré para o pós-teste, a diferença não se verificou estatisticamente significativa, sendo a magnitude do efeito moderada. Contudo, é de salientar que no grupo B/GS $(n=4)$ no préteste, há dois alunos com resultados acima do Ponte de Corte para a sua idade (Ponto de Corte=49), com valores respetivamente de 52 e 51, e como tal não têm margem de progressão para o pós- teste dado já estarem próximo do score máximo no pré-teste. Por sua vez, uma análise estatística focada nas possíveis variáveis preditivas Grupo e Idade mostra que o grupo B apresenta uma maior evolução cognitiva que o grupo A e que nos dois grupos, essa evolução é maior nas crianças de quatro anos (MS).

\section{CONCLUSIONES}

Tendo como objetivo geral, estabelecer se a relação entre os conceitos básicos de Boehm e os jogos infantis favorece 0 desenvolvimento cognitivo conclui-se que os jogos infantis são uma ferramenta útil que o professor pode aplicar nas suas aulas visando ajudar as crianças na compreensão dos conceitos básicos. No que respeita a favorecer o desenvolvimento cognitivo, é pouco conclusivo pois os resultados obtidos no pós-teste pelas crianças dos grupos A e B são muito similares, tendo em conta que o grupo B não realizou os jogos infantis. No que refere ao objetivo específico verificar se existem variáveis que influenciam a compreensão dos conceitos em crianças que frequentam a pré-escola, pode dizer-se que os resultados podem sofrer muitas influências da escola, da eficácia das práticas pedagógicas, do número de alunos, do interesse pela língua, da comunidade e dos efeitos sociais. No que concerne à exposição linguística, esta pode influenciar a compreensão dos conceitos básicos. 


\section{O DESENVOLVIMENTO DA COMPETÊNCIA BILINGUE DE CRIANCAS EM IDADE PRÉ-ESCOLAR}

Como referido anteriormente, os dois grupos em estudo apresentam características diferentes, principalmente a nível da dimensão, sendo o grupo $A(n=19)$ e 0 grupo $B(n=12)$. Algumas variáveis podem influenciar a compreensão dos conceitos básicos: 0 Sexo, a Idade, 0 Grupo e a Escolaridade. A única variável independente que se revelou preditiva do resultado no pré- teste foi a Idade, isto porque 0 grupo de 5 anos revela uma média superior $(M=20,81)$ do que as crianças com 4 anos $(M=11,93)$. A criança interfere ativamente na sua aprendizagem e é responsável pela construção do seu próprio conhecimento. 0 meio social, a escola e os inputs que vai receber de todas as suas vivências vão ajudá-la noseu desenvolvimento social e académico. A propósito da aprendizagem bilingue, Flores (2019) defende que a criança que é exposta a dois idiomas é capaz de adquiri-los "como duas línguas maternas, sem desvantagens cognitivas", acrescentando que a mente humana "está preparada para o bilinguismo". Para que essa aquisição seja possível a criança tem de ter contacto com a língua e ter necessidade de a falar e interagir, usando-a (Flores, 2019). Grosjean $(2015,2018,2021)$ propõe que a aprendizagem de uma segunda língua tem a ver com a participação da criança na sociedade. Logo, os adultos devem solicitar a criança para atividades recreativas e de comunicação onde seja necessário empregar a sua segunda língua. Estudos recentes, Grosjean (2018 e 2021), argumentam que uma pessoa pode vir a ser bilingue em qualquer idade. 0 termo bilinguismo remete para o mundo infantil, mas, segundo 0 autor uma pessoa pode aprender duas ou mais línguas a qualquer momento da sua vida seja criança, adolescente ou adulto. De acordo com o objetivo específico investigar se implementação dos jogos infantis é uma vantagem ou não para a compreensão dos conceitos básicos das crianças do pré-escolar, não se verifica uma relação significativa entre os resultados dos jogos infantis e os resultados obtidos nopré- teste, no pós-teste e na evolução sentida do pré para o pós-teste. Não é pelo facto das crianças revelarem melhores resultados nos jogos que vão revelar melhores resultados nos conceitos básicos. Apesar dos jogos não terem um papel influenciador (Piaget, 1978, p.119) são um meio através do qual as crianças começam a interagir consigo mesmas e com o mundo externo. Uma criança que tem a possibilidade de brincar, desenvolve-se cognitivamente, socialmente, afetivamente e fisicamente, assim, os jogos contribuem positivamente para o seu desenvolvimento global. Brock; Dodds; Jarvis e Olusoga (2011) referem Moyles (2002) e Hutt (s.d.) segundo os quais, a categorização da brincadeira está associada ao desenvolvimento cognitivo/intelectual, social e criativo da criança. A primeira categoria é chamada de epistémica e engloba as brincadeiras relacionadas com 0 desenvolvimento cognitivo/intelectual. A segunda categoria é chamada de lúdica e abarca as brincadeiras associadas ao desenvolvimento social e criativo das crianças. A terceira e última categoria engloba os jogos com regras. Brock; Dodds; Jarvis e Olusoga (2011) informam que Salomão; Martini e Jordão (2007) dizem que as brincadeiras e os jogos que as crianças realizam contribuem para diversos níveis de desenvolvimento como é 0 caso do desenvolvimento físico, autonomia, intelectual e social e ainda contribui positivamente para a formação da personalidade das mesmas. Sabe-se que na pré-escola, as crianças exigem uma atenção individualizada e é mais fácil dar respostas às suas solicitações em grupos mais pequenos. Por outro lado, a existência de grupos com crianças de diferentes idades acentua a diversidade e enriquece as interações no grupo, proporcionando múltiplas ocasiões de aprendizagem entre as crianças, uma característica que pode ser considerada comum tanto ao grupo A como ao grupo B. Outros fatores que podem ter influenciado os resultados são as condições institucionais, uma vez que as crianças do grupo B são na sua maioria alunos internos, isto é, aprendem o francês e 0 português na mesma escola, enquanto que todas as crianças do grupo A, são externas (originárias de várias escolas, onde aprendem o francês com práticas pedagógicas diferenciadas). Neste estudo, as crianças também revelam uma melhoria no domínio dos conceitos básicos aplicados nos jogos infantis. Os conceitos mais fáceis são os qualificados no âmbito de quantidade, espaço, tempo e outros e revelaram mais dificuldade nos conceitos "igual" e "ao redor". Quando uma criança entra no ensino pré- escolar, o desenvolvimento da língua tem de ser abordado formalmente, independentemente de a criança ter ou não idade apropriada para desenvolver esta competência. Estudos realizados, propõem que o desenvolvimento cognitivo e académico está relacionado com o contexto de imersão e com a educação bilingue (Baker \& Wright, 2021). 


\section{REFERENCIAS BIBLIOGRÁFICAS}

Aguiar, J. S. (2016). Educação Inclusiva. Jogos para o ensino de conceitos. Ed. Papirus. Abdelilah-Bauer, B. (2015). Le défi des enfants bilingues. Éditeur Hors Collection

Social.

Baker, C. Wright, W. E. (2021). Foundations of Bilingual Education and Bilingualism.

7th ed. Multilingual Matters.

Boehm, A.E. (2012). Test Boehm de Conceptos básicos 3.

Bogdan, R. \& Biklen, S. (2010). Investigação Qualitativa em Educação: Uma Introdução à Teoria e aos Métodos. Ed.: Porto Editora. ISBN 978-972-0-34112-2.

Brock, A., Dodds, S., Jarvis, P. \& Olusoga, Y. (2011). Brincar - Aprendizagem para a vida. Artmed Editora S. A.

Carmo, H. \& Ferreira, M. M. (2015). Metodologia da Investigação: Guia para autoaprendizagem (3a ed.). Ed. Universidade Aberta.

Correia, L. (2017). O efeito da exposição linguística sobre a competência lexical de crianças lusodescendentes residentes na Alemanha. Tese de Mestrado. Universidade do Minho.

Correia, L. \& Flores, C. (2017). The Role of Input Factors in the Lexical. Development of European Portuguese as a Heritage Language in Portuguese-German Bilingual Speakers. Languages, 2 (4), 30. https://doi.org/10.3390/languages2040030

Cote, L. R. \& Bornstein, M. H. (2014). Productive vocabulary among three groups of bilingual American children: Comparison and prediction. First Language, 34, 467- 485.

Flores, C. (2019). Bilinguismo Infantil: Um legado valioso do fenómeno migratório.

Diacrítica, 31(3), 237-250. https://doi.org/10.21814/diacritica.395

Hoff, E., Core, C., Place, S., Rumiche, R., Senor, M., \& Parra, M. (2012). Dual language exposure and early bilingual development. Journal of Child Language, 39, 1-27.

Gómez, G. R., Flores, J. G. \& Jiménez, E. G. (1999). Metodología de la investigación cualitativa. Ediciones Aljibe. Gonçalves, S. P., Gonçalves, P. J., \& Marques, C. G. (2021). Manual de Investigação Qualitativa (1a ed). Pactor. Grosjean, F. (2015). Paler plusieurs langues: le monde des bilangues. Albin Michel.

Grosjean, F. (2018). Être bilingue aujourd hui «Revue française de linguistique appliquée». Pub linguistiques Vol. XXIII (pp.7-14) ISSN 1386-1204.

https://www.cairn.info/revue-francaise-de-linguistique-appliquee-2018-2-page- 7.htm

Grosjean, F. (2021). Life as a Bilingual. Cambridge University Press. Edição do Kindle, p.276.

Kamii, C. (2003). A Teoria de Piaget e a Educação Pré-escolar (4a ed.). Ed.: Instituto Piaget.

Marchman, V. A., Fernald, A., \& Hurtado, N. (2010). How vocabulary size in two languages relates to efficiency in spoken word recognition by young Spanish- English bilinguals. Journal of Child Language, 37, 817-840.

Moyles, J. (2002). Só brincar? 0 papel do brincar na educação infantil. Artmed Editora S.A.

Piaget, J. (1978). 0 nascimento da inteligência na criança. Zahar.

Salomão, H.; Martini, M \& Jordão, A. (2007). A importância do lúdico na educação infantil: enfocando a brincadeira e as situações de ensino não direcionado. http://www.psicologia.pt/artigos/textos/A0358.pdf

Stake, R. K (2005). Qualitative Case Studies. In N. Denzin \& Y. Lincoln (eds). The Sage Handbook of Qualitative Research. (pp. 443-466). Sage.

Unsworth, S. (2016). Quantity and Quality of Language Input in Bilingual Language Development. In E. Nicoladis e S. Montanari (Eds.), Bilingualism Across the Lifespan (pp. 103-122). De Gruyter.

Vygotsky, L.S. (1934). A construção do pensamento e da linguagem. Martins Fontes, 2001. 
